\section{Afinal, quantos Sistemas de Informação em Saúde de base nacional existem no Brasil?}

\author{
After all, how many nationwide Health \\ Information Systems are there in Brazil?
}

\begin{abstract}
Al final, ¿cuántos Sistemas de Información en Salud de base nacional existen en Brasil?
\end{abstract}

Giliate Cardoso Coelho Neto 1

Arthur Chioro 1

\section{Resumo}

O objetivo da pesquisa foi identificar e analisar os Sistemas de Informação em Saúde (SIS) de base nacional que estiveram em funcionamento no Brasil entre os anos de 2010 e 2018, assim como compreender a estrutura de gestão e suporte de Tecnologia da Informação (TI) destes sistemas no Ministério da Saúde. É um estudo descritivo, baseado em análise documental, em que se buscou consolidar dados sobre os SIS de abrangência nacional com base em critérios de seleção referenciados ao conceito de SIS. Os documentos analisados trouxeram dados incompletos e bastante diferentes entre si, evidenciando as diferentes interpretações dos conceitos de sistemas e sistemas de informação em saúde no Ministério da Saúde. Foram encontrados 54 SIS de base nacional em funcionamento no período de 2010 a 2018 e colhidas informações sobre sete núcleos de TI descentralizados em departamentos do Ministério da Saúde, que trabalham de forma autônoma ao Departamento de Informática do SUS (DATASUS). Ao analisar suas lógicas de funcionamento foram identificados quatro grupos de SIS: um primeiro relativo ao monitoramento de eventos de relevância para a saúde pública, um segundo voltado para um maior controle e monitoramento de programas de saúde do Ministério da Saúde, um terceiro dedicado ao cadastramento geral de pessoas físicas e jurídicas e um quarto que pode ser considerado uma "nova geração" de SIS pois, apesar de serem desenvolvidos e mantidos pelo Governo Federal, se descolam de uma lógica programática verticalizada, destinando-se à racionalização administrativa e melhoria da qualidade da prestação de serviços do SUS em âmbito locorregional.

Atenção Primária à Saúde; Sistemas de Informação em Saúde; Tecnologia da Informação; Integração de Sistemas

\author{
Correspondência \\ G. C. Coelho Neto \\ Rua Rui Calaca 94, apto. 102, Recife, PE 52020-110, Brasil. \\ giliate@gmail.com \\ 1 Programa de Pós-graduação em Saúde Coletiva, Universidade \\ Federal de São Paulo, São Paulo, Brasil.
}




\section{Introdução}

Sistemas de Informação em Saúde (SIS) são instrumentos que, por meio do processamento de dados coletados em serviços de saúde e outros locais, dão suporte à produção de informações para a melhor compreensão dos problemas e tomada de decisão no âmbito das políticas e do cuidado em saúde 1 . No Brasil, quando esses sistemas têm abrangência nacional são chamados de fontes de dados nacionais sob a gestão do Sistema Único de Saúde (SUS) 2, SIS de abrangência nacional 3 ou de base nacional 4, ficando a custódia dos dados e a manutenção de software geralmente sob a responsabilidade do Ministério da Saúde.

A história dos SIS está intimamente relacionada à evolução das políticas públicas de saúde no Brasil. Moraes 5 descreve dois "blocos lógicos" de práxis institucional do Estado no desenvolvimento de sistemas e organização da informação em saúde: o primeiro estaria relacionado aos SIS que visavam a dar suporte à racionalização administrativa da assistência médico-previdenciária, regido por uma lógica contábil, quantitativa e centralizadora, que tinha o procedimento médico como principal evento básico de interesse. Um segundo bloco lógico teria relação com os SIS desenvolvidos sob a racionalidade campanhista, voltados ao monitoramento de doenças e agravos, e organização e controle de programas verticalizados.

Para além do seu aspecto funcional, a informação em saúde no último século foi inserida num contexto epistêmico de expansão do olhar do Estado e do próprio capitalismo sobre os processos, trabalhos e hábitos de saúde da população ${ }^{6}$. A multiplicação das interfaces de coleta de dados de saúde - dos formulários de papel dos SIS aos sensores automatizados - é uma das faces visíveis desse fenômeno. Nos serviços de saúde e órgãos responsáveis pela gestão do SUS em todas as esferas de governo, é comum profissionais de saúde e gestores públicos conviverem com dezenas de sistemas em seus ambientes de trabalho, que captam dados de forma redundante e acabam por gerar retrabalho, aumento de custos e incremento do risco de inconsistências nas informações produzidas 7,8. Moraes \& Gomez 9 associam a fragmentação dos SIS à própria estrutura burocrática pulverizada da máquina estatal com os setores internos do Ministério da Saúde, por meio dos chamados núcleos de Tecnologia da Informação (TI), tendo autonomia para criar e desenvolver as suas próprias soluções de software, independentemente de haver ou não diálogo com as instâncias formais do SUS e do próprio Ministério da Saúde.

Tais núcleos são descritos em documentos do Ministério da Saúde pelo menos desde 2002 10, e geralmente são constituídos por equipes de profissionais de TI contratadas com base em convênios de cooperação técnica, assumindo tarefas de desenvolvimento e/ou manutenção de soluções de softwares, baseando-se na demanda dos setores aos quais estão vinculados. Os núcleos foram criados com base em uma insuficiente resposta do Departamento de Informática do SUS (DATASUS) às demandas desses setores, mas são criticados por nem sempre seguirem as regras de segurança, integridade e disponibilidade dos produtos gerados 11 .

As políticas de controle e governança das Informações e Tecnologias da Informação em Saúde (ITIS) adotadas pelo Ministério da Saúde que, em tese, poderiam efetuar um contrapeso à tendência fragmentadora, não têm se mostrado suficientes para a indução dos governos e mercados na busca por soluções integradas. No passado recente, alguns avanços puderam ser notados, como a aprovação das políticas nacionais de informação e informática em saúde 12,13, da Resolução no 6/2013 14, da Comissão Intergestora Tripartite (CIT), que exige que todo novo SIS de base nacional seja aprovado na CIT, da Portaria no 2.073/2011 15, que define padrões nacionais de interoperabilidade, e a criação do Comitê de Informação e Informática do Ministério da Saúde 16. Todavia, baseada em normatizações infralegais e com escassos espaços de produção de consensos entre o governo e a sociedade, a política de governança das ITIS ainda tem evidente limitação de eficácia, persistindo o cenário de fragmentação.

Sob o aspecto tecnológico, a integração de SIS se materializa por meio de ferramentas que possibilitam tanto o cruzamento de diferentes bases de dados ${ }^{17}$, a unificação de componentes (Gartner IT Glossary. System Integration. https://www.gartner.com/it-glossary/system-integration, acessado em 16/Set/2019) ou a troca de dados automatizada por intermédio de mensagens e arquivos padronizados (interoperabilidade sintática) ${ }^{18}$. Sob o ponto de vista semântico, refere-se à padronização de termos e significados e o desenvolvimento de arquétipos informacionais 19. Vinculados a essas soluções, porém, existem atores políticos com seus interesses e projetos que disputam não só a adoção 
de tecnologias e padrões e modos de fazer junto ao Estado, mas o próprio papel no Estado na relação público-privado, com impacto direto na capacidade de resposta do setor estatal aos problemas de saúde da população. Ou seja, qualquer projeto de integração de SIS tem um forte componente técnico-político e exige a compreensão não só dos sistemas envolvidos, mas também das estruturas, relações de poder e processos de gestão associados - elementos centrais na governança das ITIS 20.

Assim como a incorporação de TI no setor de saúde de uma forma geral, ainda é incerto que tais integrações resultem em redução de custo 21,22, porém, alguns estudos vêm apontando possíveis benefícios econômicos com a integração e a troca de informações entre os SIS. No Reino Unido, as organizações responsáveis pela gestão locorregional dos serviços de saúde no Sistema Nacional de Saúde (NHS Trusts - National Health Service), relataram uma diminuição de custos entre 3\% e 4\% decorrente de ações integradas de interoperabilidade e análise de grandes volumes de dados 23 . Na Coreia do Sul, constatou-se uma diminuição de $13 \%$ dos gastos relacionados com cuidados em saúde quando comparadas as equipes que dispunham de troca de informações clínicas e as que não possuíam 24.

Qualquer agenda ou projeto de integração de SIS tem como premissa a definição sobre quais Sistemas serão objeto desta integração. Um melhor conhecimento sobre o portfólio dos SIS de base nacional pode contribuir não somente com a eficácia dos processos de integração, mas também apoiar a produção de pesquisas acadêmicas e o desenvolvimento das políticas de governança das ITIS na esfera nacional do SUS. Ao mesmo tempo, compreender melhor a estrutura de gestão relacionada a esse portfólio é relevante para entender a sua evolução histórica, incluindo aí a persistente fragmentação de sistemas.

Quando apontamos o olhar para os grandes SIS em funcionamento no Brasil, entretanto, persiste a interrogação: quantos e quais SIS de base nacional estão em funcionamento no país? Aparentemente simples, a resposta a essa questão não é consensuada nos documentos e discursos oficiais, nem na literatura especializada. O site oficial do DATASUS (http://www2.datasus.gov.br/DATASUS/ index.php), por exemplo, apresentava, em setembro de 2019, uma lista com 37 sistemas no menu da página inicial e outra lista com 101 "sistemas de saúde" em página secundária (Sistemas e Aplicativos DATASUS. http://datasus.saude.gov.br/sistemas-e-aplicativos, acessado em 06/Set/2019). Já o Plano Diretor de Tecnologia da Informação (PDTI) do Ministério da Saúde referente ao período 20142015 continha uma lista de 190 sistemas em funcionamento 11. No XXXIII Congresso Nacional de Secretarias Municipais de Saúde, em 2017, a diretoria do DATASUS relatou um total 667 sistemas no Ministério da Saúde 25.

A literatura especializada contempla poucos estudos e também não indica um número definitivo. Uma publicação conjunta da Organização Pan-Americana da Saúde (OPAS), Ministério da Saúde e Fundação Oswaldo Cruz (Fiocruz), o livro A Experiência Brasileira em Sistemas de Informação em Saúde 3 se propôs a debater apenas quatro SIS de base nacional. Numa abordagem mais ampla, Moraes 2 (p. 653) cita as "principais fontes de dados nacionais sob a gestão do SUS nas três esferas de governo".

Considerando isso, o objetivo do estudo foi identificar e analisar os SIS de base nacional que estiveram em funcionamento no Brasil entre os anos de 2010 e 2018, assim como compreender a estrutura de gestão e suporte de TI destes sistemas no Ministério da Saúde.

\section{Métodos}

É um estudo descritivo, baseado em análise documental, em que buscou-se consolidar evidências de SIS de base nacional que estiveram em funcionamento em algum período entre os anos de 2010 a 2018. A amplitude do recorte temporal possibilitou que um conjunto de documentos fosse incluído na pesquisa, o que mostrou-se fundamental haja vista a escassez de registros oficiais sobre o tema.

O estudo iniciou com uma revisão da literatura que se estendeu durante todas as etapas da pesquisa, entre março de 2017 a outubro de 2018. Foram realizadas múltiplas buscas booleanas nas bases LILACS, MEDLINE e SciELO, além da plataforma Sucupira da Coordenação de Aperfeiçoamento de Pessoal de Nível Superior (Capes), utilizando-se os seguintes descritores: Sistemas de Informação em Saúde, Informática em Saúde, Tecnologia da Informação, Informática em Saúde Pública e Política de Saúde. Foram também consultados os principais tratados de Saúde Coletiva brasileiros. 
$\mathrm{Na}$ primeira etapa da análise documental recuperou-se documentos relacionados à política de Informação e Informática do Ministério da Saúde. Foram analisados os PDTI dos anos de 2014-2015, 2016 e 2017-2018, a Política Nacional de Informação e Informática em Saúde (PNIIS), publicada em 2016, e o Relatório de Gestão do DATASUS 2011-2014. Também foram recuperados três termos de referência vinculados a processos de contratação de empresas de software, dois cujos objetos estavam relacionados à manutenção dos sistemas do Ministério da Saúde (Editais do Pregão Eletrônico no 154/2010 e do Contrato no 22/2013) e outro do Programa de Informação das Unidades Básicas de Saúde (PIUBS - Edital no 01/2017). Todos esses documentos foram encontrados nos sites oficiais do Governo Federal.

Numa segunda etapa, buscou-se obter informações acerca dos sistemas em funcionamento no Ministério da Saúde com base em pedidos formais realizados ao Ministério por meio do Serviço de Informação ao Cidadão (Lei no 12.527/201126). A Lei de Acesso à Informação (LAI), como é conhecida, estabelece que qualquer documento ou informação sob posse do governo pode ser solicitada e/ ou acessada por um cidadão, a não ser aquelas formalmente classificadas como reservadas (prazo de 5 anos de sigilo), secretas (15 anos de sigilo) e ultrassecretas (25 anos de sigilo). Pela lei, os órgãos públicos ficam obrigados a criar Serviços de Informação ao Cidadão (SIC) para receber os pedidos de informação. A partir da data de formalização desses pedidos, o órgão tem o prazo de 20 dias para emitir a resposta, podendo prorrogá-lo por mais 10 dias. Caso o cidadão não se sinta contemplado com a resposta, há a possibilidade de se recorrer a instâncias superiores. No Governo Federal, existe um sistema específico, o e-SIC, para registrar e acompanhar os pedidos de informação, e que pode ser acessado pelo endereço: https://esic.cgu.gov.br.

A definição dos setores internos destinatários dos pedidos de informação foi construída com base em anotações pessoais de um dos autores, durante o período em que ocupou cargo de chefia na área de TI do Ministério da Saúde, que continha registros de quais departamentos tinham Núcleos de TI em funcionamento, haja vista a ausência de informações detalhadas sobre tais núcleos nos documentos oficiais. Todos os pedidos foram respondidos no prazo previsto em lei e apenas em um deles houve a necessidade de se recorrer a instâncias superiores do Ministério da Saúde, devido à incompletude da resposta. Ao DATASUS foi solicitada a lista dos atuais sistemas de informação mantidos pelo departamento e que estavam em produção, inclusive os que não estavam como prioridade do PDTI, assim como a lista dos "Núcleos Informais de TI" em funcionamento no Ministério da Saúde. Aos Núcleos de TI dos departamentos foram solicitadas a lista dos sistemas de informação sob sua responsabilidade, a lista de membros da equipe, as parcerias externas para o desenvolvimento de software ou outros serviços de TI e o ano de início das atividades. O conteúdo dos pedidos pode ser acessado de forma aberta no sistema e-SIC por meio dos seguintes protocolos: 25820004082201795, 25820003308201811, 25820005768201884, 25820005770201853, 25820005771201806, 25820005772201842, 25820005773201897, 25820005774201831, 25820005775201886, 25820005776201821.

$\mathrm{Na}$ terceira etapa foram realizadas buscas de documentos na Internet usando-se o buscador Google em sites oficiais do Ministério da Saúde. Para isso, utilizou-se as combinações "sistemas" e "datasus", "sistemas de informação" e "datasus”, assim como o termo "sistemas de informação". As buscas foram restringidas a três endereços oficiais do Ministério da Saúde na Internet: https://datasus. saude.gov.br/, https://datasus1.saude.gov.br/, https://www.saude.gov.br/, bem como a arquivos em formato PDF por meio de mecanismos do próprio buscador. Foram encontrados 1.898 resultados de busca no total, que foram analisados em busca de pistas sobre SIS em funcionamento no período entre 2010 e 2018 .

\section{Resultados}

Os documentos analisados trouxeram dados incompletos e bastante diferentes entre si, conforme disposto no Quadro 1.

A significativa discrepância entre as fontes pode ser explicada em parte pelas diferentes interpretações dos conceitos de sistemas e sistemas de informação de saúde, decorrentes da ausência de definição única no âmbito no Ministério da Saúde, e que assumiu os seguintes significados nos documentos analisados: 


\section{Quadro 1}

Comparativo entre a informação sobre quantidade e listas de sistemas de documentos, arquivos e sites oficiais do Ministério da Saúde.

\begin{tabular}{|c|c|c|}
\hline FONTE & QUANTIFICA SIS? & LISTA NOMINALMENTE OS SIS? \\
\hline $\begin{array}{l}\text { Edital de Pregão Eletrônico no 154/2010 da Secretaria Executiva do } \\
\text { Ministério da Saúde }\end{array}$ & Sim (49 sistemas) & $\operatorname{Sim}$ \\
\hline PDTI 2014-2015 & Sim (129 sistemas) & Sim \\
\hline PDTI 2016 & Não & Não \\
\hline PDTI 2017-2018 & Não & Não \\
\hline Relatório Executivo de Gestão do DATASUS do período 2011-2014 & Sim (390 sistemas) & Sim (64 sistemas) \\
\hline $\begin{array}{l}\text { Política Nacional de Informação e Informática (PNIIS) } \\
\text { (Portaria no 389/2015) }\end{array}$ & Não & Não \\
\hline $\begin{array}{l}\text { Termo de Referência para a contratação de Fábrica de software (contrato } \\
\text { 22/2013) }\end{array}$ & Sim (31 sistemas) & Sim \\
\hline $\begin{array}{l}\text { Resposta } 01 \text { do Ministério da Saúde (LAl) - Protocolo } \\
25820004082201795\end{array}$ & Sim (465 sistemas) & $\begin{array}{c}\text { Parcial (diversos links em que não é } \\
\text { possível identificar os sistemas) }\end{array}$ \\
\hline $\begin{array}{l}\text { Resposta } 02 \text { do Ministério da Saúde (LAl) - Protocolo } \\
25820004082201795\end{array}$ & Sim (67 sistemas) & Sim \\
\hline Site do DATASUS & Sim (37 sistemas) & Sim \\
\hline Termo de Referência do PIUBS (Apenas SIS em uso na Atenção Básica) & Sim (29 sistemas) & Sim \\
\hline Portal Tabnet/DATASUS & Sim (8 sistemas) & Sim \\
\hline
\end{tabular}

DATASUS: Departamento de Informática do SUS; LAI: Lei de Acesso à Informação; PDTI: Plano Diretor de Tecnologia da Informação; PIUBS: Programa de Informação das Unidades Básicas de Saúde; SIS: Sistemas de Informação à Saúde.

(1) Instalações genéricas de software nos datacenters, incluindo sistemas de informação em saúde, ferramentas de infraestrutura de hardware, sites e instalações de software múltiplas. Numa das respostas obtidas junto ao Ministério da Saúde, por exemplo, foi enviada uma lista com todos os softwares instalados na infraestrutura do DATASUS em Brasília, incluindo ferramentas de otimização de bancos de dados, monitoramento de redes etc., assim como instalações múltiplas do mesmo sistema (o SISREG Sistema Nacional de Regulação, por exemplo, tinha nove instalações regionalizadas);

(2) Softwares únicos adquiridos ou desenvolvidos pelo Ministério da Saúde, incluindo os sistemas administrativos internos e os SIS externos (de base nacional ou não);

(3) Sistemas prioritários definidos de forma discricionária pela instituição para fins específicos, como, por exemplo, os SIS listados nos editais para a contratação de manutenção de sistemas por fábrica de software.

Em nenhum dos documentos analisados havia uma lista de SIS, em consonância com a definição citada no primeiro parágrafo deste artigo ou com outra definição, seja de base nacional ou não. Para se chegar nessa relação foi necessário inicialmente analisar os dados de todos os documentos coletados, excluindo-se as duplicidades e formando uma lista unificada. Com isso, foram visualizados 429 registros possíveis de soluções únicas ou projetos de software no portfólio do Ministério da Saúde.

Em seguida, foi realizado um breve estudo sobre cada uma dessas, sendo executada uma tentativa de acesso via web (baseada nos endereços web disponibilizados) e procura na Internet por documentação técnica ou informativa.

Como critérios de inclusão, considerou-se a presença de interface de captação de dados utilizada por estados e municípios no período de 2010 a 2018 e o armazenamento dos dados em bases centralizadas no Ministério da Saúde. Como critérios de exclusão, usou-se: projetos de software não concluídos, sistemas administrativos internos, sistemas que não tinham relação direta com secretarias de saúde, sistemas em que não foi identificada interface de captação de dados com estados e municípios, ferramentas de Business Inteligence, sites informativos, módulos acessórios de sistemas, webservices, ferramentas de transmissão de dados e sistemas descontinuados antes de 2010. Chegou-se a uma lista final de 54 SIS de base nacional, descritos no Quadro 2. 
Quadro 2

Sistemas de Informação em Saúde (SIS) de base nacional em funcionamento no Brasil entre 2010 e 2018.

\begin{tabular}{|c|c|c|}
\hline SIGLA & SISTEMA & DESCRIÇÃO \\
\hline AMAQ & $\begin{array}{l}\text { Autoavaliação para Melhoria do Acesso e da Qualidade } \\
\text { da Atenção Básica }\end{array}$ & $\begin{array}{l}\text { Avaliação do processo de trabalho da atenção primária } \\
\text { à saúde }\end{array}$ \\
\hline APURASUS & Sistema de Apuração e Gestão de Custos do SUS & $\begin{array}{l}\text { Apuração e gestão de custos em unidades de saúde } \\
\text { do SUS }\end{array}$ \\
\hline BFA & Sistema do Programa Bolsa Família na Saúde & $\begin{array}{c}\text { Controle e monitoramento da antropometria de } \\
\text { beneficiários do Programa Bolsa família }\end{array}$ \\
\hline BLH-WEB & $\begin{array}{l}\text { Sistema de Gerenciamento e Produção de Bancos de } \\
\text { Leite Humano }\end{array}$ & Apoio ao gerenciamento de Bancos de Leite Humano \\
\hline BPS & Banco de Preços em Saúde & $\begin{array}{c}\text { Registro e disponibilização das informações das compras } \\
\text { públicas e privadas de medicamentos e produtos } \\
\text { para a saúde }\end{array}$ \\
\hline CADSUS & Sistema de Cadastramento dos Usuários do SUS & Cadastro dos usuários do SUS \\
\hline CMD & Conjunto Mínimo de Dados & $\begin{array}{l}\text { Registro de contatos assistenciais em estabelecimentos } \\
\text { de saúde }\end{array}$ \\
\hline CNES & Cadastro Nacional dos Estabelecimentos de Saúde & Cadastro de estabelecimentos de saúde \\
\hline COAGULOPATIAS WEB & Hemovida web - Coagulopatias & $\begin{array}{l}\text { Controle e monitoramento de pacientes com } \\
\text { coagulopatias, incluindo o quantitativo de fatores de } \\
\text { coagulação dispensados. }\end{array}$ \\
\hline e-SUS AB/SISAB & Sistema de Informação em Saúde para a Atenção Básica & $\begin{array}{l}\text { Apoio à gestão do cuidado e controle e monitoramento } \\
\text { das atividades e procedimentos realizados na atenção } \\
\text { primária à saúde }\end{array}$ \\
\hline FIQUE SABENDO & $\begin{array}{c}\text { Sistema de controle dos Testes Rápidos de HIV, Sífilis e } \\
\text { Hepatites Virais para ações estratégicas. }\end{array}$ & Controle logístico de testes rápidos \\
\hline GAL & Sistema de Gestão de Ambiente Laboratorial & $\begin{array}{c}\text { Controle e monitoramento de resultados laboratoriais de } \\
\text { doenças e agravos de interesse da saúde pública; } \\
\text { Apoio a gerenciamento de laboratórios estaduais } \\
\text { de saúde pública }\end{array}$ \\
\hline $\begin{array}{l}\text { HEMOGLOBINOPATIAS } \\
\text { WEB }\end{array}$ & Hemovida Web - doença falciforme - HWDF & $\begin{array}{l}\text { Controle e monitoramento de pacientes com doença } \\
\text { falciforme e outras hemoglobinopatias }\end{array}$ \\
\hline HIPERDIA & $\begin{array}{c}\text { Sistema de Cadastramento e Acompanhamento de } \\
\text { Hipertensos e Diabéticos }\end{array}$ & $\begin{array}{l}\text { Controle e monitoramento de pacientes com } \\
\text { hipertensão e diabetes }\end{array}$ \\
\hline HÓRUS BÁSICO & Sistema Nacional de Assistência Farmacêutica & $\begin{array}{l}\text { Controle logístico de medicamentos do componente } \\
\text { básico da Política Nacional de Assistência Farmacêutica }\end{array}$ \\
\hline HÓRUS ESPECIALIZADO & Sistema Nacional de Assistência Farmacêutica & $\begin{array}{c}\text { Controle logístico de medicamentos do componente } \\
\text { especializado da Política Nacional } \\
\text { de Assistência Farmacêutica }\end{array}$ \\
\hline HÓRUS ESTRATÉGICO & Sistema Nacional de Assistência Farmacêutica & $\begin{array}{c}\text { Controle logístico de medicamentos do componente } \\
\text { estratégico da Política Nacional de } \\
\text { Assistência Farmacêutica }\end{array}$ \\
\hline PAINEL-PSE & Sistema de Adesão ao Programa Saúde na Escola & $\begin{array}{c}\text { Controle e monitoramento de atividades realizadas pelas } \\
\text { equipes de atenção básica no âmbito do } \\
\text { Programa Saúde na Escola }\end{array}$ \\
\hline PMAQ-AB & $\begin{array}{l}\text { Programa Nacional de Melhoria do Acesso e da } \\
\text { Qualidade da Atenção Básica }\end{array}$ & $\begin{array}{c}\text { Controle, monitoramento e avaliação de ações } \\
\text { programáticas e processos } \\
\text { de trabalho da APS }\end{array}$ \\
\hline
\end{tabular}

(continua) 
Quadro 2 (continuação)

\begin{tabular}{|c|c|c|}
\hline SIGLA & SISTEMA & DESCRIÇÃO \\
\hline PROGRAMASUS & Sistema de programações de ações e serviços de saúde & $\begin{array}{l}\text { Registro dos quantitativos físicos e financeiros das ações } \\
\text { e serviços de saúde a serem desenvolvidas no âmbito } \\
\text { regional, decorrente de pactuação entre gestores. }\end{array}$ \\
\hline RAAS-AD & Registro das Ações de Saúde da Atenção Domiciliar & $\begin{array}{c}\text { Controle e monitoramento das ações e procedimentos } \\
\text { da atenção domiciliar no âmbito do } \\
\text { Programa Melhor em Casa }\end{array}$ \\
\hline RESP & Resposta a Eventos de Saúde Pública & $\begin{array}{l}\text { Notificação, controle e monitoramento de casos } \\
\text { suspeitos ou confirmados de microcefalia }\end{array}$ \\
\hline SARGSUS & Sistema de Apoio à Elaboração do Relatório de Gestão & $\begin{array}{l}\text { Apoio à gestão municipal para elaboração e envio do } \\
\text { Relatório Anual de Gestão (RAG) ao Conselho de Saúde }\end{array}$ \\
\hline SI-PNI & $\begin{array}{l}\text { Sistema de informação do Programa Nacional de } \\
\text { Imunização }\end{array}$ & $\begin{array}{l}\text { Controle logístico de imunobiológicos e monitoramento } \\
\text { da cobertura vacinal }\end{array}$ \\
\hline SIA & Sistema de Informações Ambulatoriais & $\begin{array}{l}\text { Controle e monitoramento da produção de } \\
\text { procedimentos ambulatoriais }\end{array}$ \\
\hline SIAB & Sistema de Informação da Atenção Básica & $\begin{array}{l}\text { Controle e monitoramento das atividades e } \\
\text { procedimentos realizados na Atenção Primária à Saúde }\end{array}$ \\
\hline SIACS & Sistema de Acompanhamento dos Conselhos de Saúde & $\begin{array}{c}\text { Registro de dados sobre os conselhos de saúde de todo } \\
\text { país - conselheiros, composição e cumprimento de } \\
\text { normas legais }\end{array}$ \\
\hline SIASI & Sistema de Informação da Saúde Indígena & $\begin{array}{c}\text { Controle e monitoramento de informações demográficas } \\
\text { e da atenção à saúde dos povos indígenas }\end{array}$ \\
\hline SICLOM & $\begin{array}{l}\text { Sistema de controle e monitoramento clínico e } \\
\text { laboratorial dos pacientes em tratamento de hepatite }\end{array}$ & Controle logístico dos medicamentos antirretrovirais \\
\hline SIES & Sistema de Informação de Insumos Estratégicos & Controle logístico de insumos estratégicos \\
\hline SIM & Sistema de Informações sobre Mortalidade & Controle e monitoramento de óbitos \\
\hline SINAN NET & Sistema de Informação de Agravos de Notificação & $\begin{array}{l}\text { Notificação, controle e monitoramento de casos de } \\
\text { doenças e agravos de interesse da saúde pública }\end{array}$ \\
\hline $\begin{array}{l}\text { SINAN DENGUE/ } \\
\text { CHIKUNGUNYA }\end{array}$ & $\begin{array}{c}\text { Sistema de Informação de Agravos de Notificação para } \\
\text { Dengue e Chikungunya }\end{array}$ & $\begin{array}{c}\text { Notificação, controle e monitoramento de casos de } \\
\text { dengue e febre chikungunya }\end{array}$ \\
\hline SINAN INFLUENZA & $\begin{array}{l}\text { Sistema de Informação de Agravos e Notificação - } \\
\text { Influenza }\end{array}$ & $\begin{array}{l}\text { Notificação, controle e monitoramento de casos de } \\
\text { síndrome respiratória aguda grave }\end{array}$ \\
\hline SINASC & Sistema de Informações sobre Nascidos Vivos & Controle e monitoramento de óbitos \\
\hline SIOPS & $\begin{array}{l}\text { Sistema de Informações sobre Orçamentos } \\
\text { Públicos em Saúde }\end{array}$ & $\begin{array}{l}\text { Controle e monitoramento de dados referentes às } \\
\text { receitas totais e às despesas com saúde dos orçamentos } \\
\text { públicos em saúde }\end{array}$ \\
\hline SIRAM & $\begin{array}{l}\text { Sistema de Registro de Atendimento à Crianças } \\
\text { com Microcefalia }\end{array}$ & $\begin{array}{l}\text { Controle e monitoramento da assistência à saúde de } \\
\text { crianças com diagnóstico de microcefalia }\end{array}$ \\
\hline SIS PRÉ-NATAL & $\begin{array}{l}\text { Sistema de Informações de Monitoramento e Avaliação } \\
\text { do Pré-Natal, Parto, Puerpério e Criança }\end{array}$ & $\begin{array}{l}\text { Controle e monitoramento da assistência à saúde da } \\
\text { gestante, puérperas e recém-nascidos }\end{array}$ \\
\hline SIS-AGUA & $\begin{array}{l}\text { Sistema de Informação de Vigilância da Qualidade da } \\
\text { Água para Consumo Humano }\end{array}$ & $\begin{array}{l}\text { Controle e monitoramento do tratamento de água } \\
\text { empregado nos sistemas e soluções alternativas de } \\
\text { abastecimento de água para consumo humano. }\end{array}$ \\
\hline SIS-CNRAC & Central Nacional de Regulação de Alta Complexidade & $\begin{array}{l}\text { Apoio à regulação assistencial de procedimentos da alta } \\
\text { complexidade no âmbito interestadual }\end{array}$ \\
\hline SIS-SOLO & $\begin{array}{l}\text { Sistema de Informação de Vigilância em Saúde de } \\
\text { Populações Expostas a Solo Contaminado }\end{array}$ & $\begin{array}{l}\text { Controle e monitoramento da saúde das populações de } \\
\text { áreas contaminadas }\end{array}$ \\
\hline
\end{tabular}

(continua) 
Quadro 2 (continuação)

\begin{tabular}{|c|c|c|}
\hline SIGLA & SISTEMA & DESCRIÇÃO \\
\hline SISCAN & Sistema de Informação do Câncer & $\begin{array}{l}\text { Controle e monitoramento das ações relacionadas à } \\
\text { detecção precoce, à confirmação diagnóstica e ao início } \\
\text { do tratamento de neoplasias malignas }\end{array}$ \\
\hline SISCEL & $\begin{array}{l}\text { Sistema de Controle de Exames Laboratoriais de CD4 } \\
\text { e Carga Viral }\end{array}$ & $\begin{array}{c}\text { Controle e monitoramento de resultados dos testes para } \\
\text { contagem de linfócitos TCD4+/TCD8+ e quantificação } \\
\text { viral do HIV }\end{array}$ \\
\hline SISGENO & Sistema de Controle de Exame de Genotipagem & $\begin{array}{l}\text { Controle e monitoramento de exames de Genotipagem } \\
\text { de HIV-1 }\end{array}$ \\
\hline SISNEO & Sistema Nacional de Triagem Pré-Natal & $\begin{array}{c}\text { Controle e monitoramento de fluxo laboratorial e } \\
\text { tratamento no âmbito do Programa nacional } \\
\text { de Triagem neonatal }\end{array}$ \\
\hline SIS-PNCD & Sistema do Programa Nacional de Controle da Dengue & $\begin{array}{l}\text { Controle e monitoramento de ações } \\
\text { de combate à dengue }\end{array}$ \\
\hline SISREG & Sistema Nacional de Regulação & $\begin{array}{l}\text { Apoio à regulação assistencial em } \\
\text { nível municipal ou estadual }\end{array}$ \\
\hline $\begin{array}{l}\text { SISTEMA DE } \\
\text { MICRONUTRIENTES }\end{array}$ & Sistema de Micronutrientes & $\begin{array}{l}\text { Controle e monitoramento da suplementação de } \\
\text { vitamina A, ferro e micronutrientes em pó na APS }\end{array}$ \\
\hline SISVAN & Sistema de Vigilância e Acompanhamento Nutricional & $\begin{array}{l}\text { Controle e monitoramento do estado nutricional e } \\
\text { consumo alimentar na APS }\end{array}$ \\
\hline SIVEP DDA & $\begin{array}{c}\text { Sistema Informatizado de Vigilância Epidemiológica de } \\
\text { Doenças Diarreicas Agudas }\end{array}$ & $\begin{array}{l}\text { Notificação, controle e monitoramento de pessoas com } \\
\text { doença diarreia aguda }\end{array}$ \\
\hline SIVEP MALÁRIA & $\begin{array}{l}\text { Sistema de Informação de Vigilância Epidemiológica de } \\
\text { notificação de casos de malária }\end{array}$ & $\begin{array}{c}\text { Notificação, controle e monitoramento } \\
\text { de casos de malária } \\
\end{array}$ \\
\hline SNT & Sistema Nacional de Transplantes & $\begin{array}{l}\text { Apoio ao gerenciamento da lista de espera de } \\
\text { transplantes no Brasil }\end{array}$ \\
\hline TELESSAÚDE/SMART & $\begin{array}{c}\text { Sistema de Monitoramento e Avaliação dos Resultados } \\
\text { do Programa Telessaúde }\end{array}$ & $\begin{array}{l}\text { Controle, monitoramento e avaliação de } \\
\text { programas de telessaúde }\end{array}$ \\
\hline VETORES MALÁRIA & $\begin{array}{l}\text { Sistema de Informação para o Controle } \\
\text { da Malária no Brasil }\end{array}$ & $\begin{array}{l}\text { Controle e monitoramento dos vetores } \\
\text { de malária no Brasil }\end{array}$ \\
\hline
\end{tabular}

APS: atenção primária à saúde; SUS: Sistema Único de Saúde; UBS: unidades básica de saúde.

Nota: a partir de 2019, e-SUS Atenção Básica (e-SUS AB) passou a se chamar e-SUS Atenção Primária à Saúde (e-SUS APS).

Nem todos esses SIS podem estar em funcionamento atualmente, haja vista a possibilidade de integração total entre alguns deles ou mesmo a decisão pela descontinuidade pela instância gestora responsável. Todavia, é importante pontuar que as bases de dados construídas valendo-se de SIS que se tornaram obsoletos devem ser preservadas para fins de análise, pesquisa, auditoria ou simplesmente registro histórico.

Em relação à estrutura de gestão de TI que dá suporte aos SIS, o Ministério da Saúde encaminhou o pedido para o DATASUS, que emitiu a seguinte resposta:

"Conforme citado no segundo parágrafo do item 5.4 do PDTI 2017-2018 DATASUS, os Núcleos Informais de TI no Ministério da Saúde atuam geralmente de forma independente e isolada do Datasus, desta forma, este departamento não mantém acompanhamento e nem controle sobre as equipes de trabalho e sistemas de informação sob a responsabilidade ou gestão de cada núcleo informal, de tal forma que não tem como fornecer as informações solicitadas" (grifo dos autores).

Diante da constatação de que o funcionamento dos núcleos não era monitorado pelo DATASUS, foram realizados novos pedidos de informação diretamente para as secretarias e departamentos do Ministério da Saúde, que responderam dentro do prazo estipulado pela LAI e trouxeram maior detalhamento sobre os "Núcleos de TI". 
O Núcleo de Tecnologia da Informação (NTI) do Departamento de Atenção Básica (DAB) iniciou suas atividades em 2010, conta com 20 profissionais de TI e é responsável pela gestão de 13 sistemas, além do portal do $\mathrm{DAB}$, tendo à época infraestrutura própria de TI para armazenamento de dados e instalações de alguns sistemas. Existia ainda uma parceria formal com a Universidade Federal de Santa Catarina (UFSC) para o desenvolvimento de software que incluía, por exemplo, a manutenção e a evolução dos sistemas da Estratégia e-SUS Atenção Primária (e-SUS APS).

O Núcleo de TI do Departamento de Vigilância, Prevenção e Controle das Infecções Sexualmente Transmissíveis, do HIV/Aids e das Hepatites Virais (DIAHV) iniciou suas atividades em 1994, tinha 29 profissionais de TI e era responsável pela gestão de 25 sistemas. O Núcleo tinha infraestrutura própria de TI com servidores, storages e unidades de backup. Não foram relatadas parcerias com instituições externas.

Na Secretaria de Gestão do Trabalho e Educação em Saúde (SGTES), o Departamento de Gestão de Educação na Saúde (DGES) tinha três profissionais em seu núcleo de TI e parceria com a Universidade Federal do Rio Grande do Norte (UFRN) para o desenvolvimento e a manutenção de oito sistemas. Já o núcleo do Departamento de Planejamento e Regulação da Provisão de Profissionais de Saúde (DPREPS) tinha cinco profissionais responsáveis por dois sistemas.

O Departamento de Regulação, Avaliação e Controle de Sistemas (DRAC) que, como o DAB, pertencia à Secretaria de Atenção à Saúde (SAS), respondeu ter apenas um profissional que fazia a gestão do Sistema de Controle do Limite Financeiro da Média e Alta Complexidade (SISMAC). Os outros sistemas do departamento eram mantidos pela equipe do DATASUS no Rio de Janeiro. O Departamento de Ações Programáticas Estratégicas (DAPES), também vinculado à SAS, afirmou não ter equipe de TI constituída, contava apenas em sua assessoria com profissionais responsáveis por produzir informações baseando-se em ferramentas disponibilizadas pelo DATASUS.

A Secretaria Especial de Saúde Indígena (SESAI) afirmou ter quatro sistemas sob a sua gestão e uma equipe formada por quatro servidores públicos e "outros colaboradores contratados".

Em sua resposta, o Fundo Nacional de Saúde (FNS) questionou o termo "Ilha/Núcleo Informal de TI", afirmando que conta com uma coordenação formal de sustentação de tecnologia da informação ligada à secretaria-executiva do FNS, sob a responsabilidade de dois servidores públicos e "colaboradores contratados por empresa licitada para o desenvolvimento e manutenção de TI, de responsabilidade do DATASUS".

O Departamento de Monitoramento e Avaliação do SUS (DEMAS), vinculado à Secretaria Executiva, afirmou que "não tem competência na área de Tecnologia da Informação e Comunicação (TIC). Esta é do DATASUS. Infraestrutura, serviços e sistemas de TIC em uso no DEMAS são, portanto, providos pelo DATASUS". A resposta é passível de questionamentos, haja vista o papel protagonista do DEMAS em atividades relacionados à gestão das ITIS no Ministério da Saúde, como a coordenação do Comitê Gestor do Programa de Informatização das UBS (PIUBS) (Portaria no 2.920, de 31 de outubro de 2017) 27 e a manutenção da Sala de Gestão Estratégica do Ministério da Saúde (http://sage.saude.gov.br).

A maioria dos 54 SIS de base nacional pode ser dividida em dois grandes grupos, de acordo com os seus principais sentidos de funcionamento, ao encontro da classificação de blocos lógicos sugerida por Moraes 2. Um primeiro grupo é composto pelos SIS que se destinam ao monitoramento de eventos de relevância para a saúde pública, como a notificação de agravos e doenças, de nascimentos e óbitos na população. Chama a atenção a fragmentação dos sistemas de notificação, que têm fluxos de dados similares e estão sob a gestão da mesma área interna do Ministério da Saúde, a Secretaria de Vigilância à Saúde - SVS). Um segundo conjunto de SIS é composto por aqueles voltados para um maior controle e monitoramento de programas de saúde do Ministério da Saúde, vinculados ou não a repasses financeiros federais.

Foi possível identificar ainda outros dois grupos de SIS. Um deles tem como principal função o cadastramento da população em geral, dos profissionais e serviços de saúde. O outro pode ser considerado uma "nova geração" de SIS que, apesar de ser desenvolvido e mantido pelo Governo Federal, descola da lógica programática verticalizada e é destinado à racionalização administrativa e melhoria da qualidade da prestação de serviços do SUS em âmbito locorregional. Nesse sentido, é possível constatar uma discreta mudança na relação da União com os entes federados, propondo-se a realizar um apoio técnico-político no campo da tecnologia da informação desvinculado de interesses diretos em obtenção de dados para fins de controle de alguma política ou programa federal (Quadro 3). 
Quadro 3

Sistemas de Informação em Saúde de base nacional entre 2010 e 2018, de acordo com a sua principal lógica de funcionamento.

\begin{tabular}{|l|c|c|}
\hline PRINCIPAL LÓGICA DE FUNCIONAMENTO & N & LISTA DE SIS \\
\hline Cadastramento populacional e de estabelecimentos de saúde & 2 & CADSUS, CNES \\
\hline Monitoramento de eventos relevantes para saúde pública & 14 & $\begin{array}{c}\text { SINAN NET, SIVEP Malária, SIVEP DDA, RESP, SINAN Dengue/ } \\
\text { Chikungunya, SINAN Influenza, SINASC, SIM, GAL, COAGULOPATIAS web, } \\
\text { HEMOGLOBINOPATIAS web, SIS-Água, SIS-Solo, VETORES-MALÁRIA }\end{array}$ \\
\hline Controle de Programas e/ou repasses Federais & 26 & $\begin{array}{c}\text { Programa Bolsa Família, PAINEL-PSE, HIPERDIA, SIRAM, SI-PNI, SIS Pré- } \\
\text { Natal, SISVAN, SIA, SIH, CMD, Fique Sabendo, SICLOM, SISCEL, SISCAN, } \\
\text { PMAQ-AB, AMAQ, RAAS-AD, SIAB, SIASI, SISGENO, ProgramaSUS, SIACS, } \\
\text { SIES, SISNEO, SIS-PNDC, Sistema de Micronutrientes }\end{array}$ \\
\hline Apoio à informatização de políticas, processos e serviços do SUS & 12 & $\begin{array}{c}\text { SISREG, HÓRUS Básico, HÓRUS Especializado, HÓRUS Estratégico, } \\
\text { TELESSAÚDE/SMART, APURASUS, BHL-WEB, BPS, SARGSUS, SNT, E-SUS } \\
\text { AB/SISAB, SIS-CNRAC }\end{array}$ \\
\hline Total & 54 & \\
\hline
\end{tabular}

AMAQ: Autoavaliação para Melhoria do Acesso e da Qualidade da Atenção Básica; APURASUS: Sistema de Apuração e Gestão de Custos do SUS; BPS: Sistema BHL-WEB: Sistema de Gerenciamento e Produção de Bancos de Leite Humano; BPS: Banco de Preços em Saúde; CMD: Conjunto Mínimo de Dados; e-SUS AB/SIAB: Sistema de Informação em Saúde para a Atenção Básica; GAL: Sistema de Gestão de Ambiente Laboratorial; HÓRUS: Sistema Nacional de Assistência Farmacêutica; PMAQ-AB: Programa Nacional de Melhoria do Acesso e da Qualidade da Atenção Básica; ProgramaSUS: Sistema de Programações de Ações e Serviços de Saúde; RAAS-AD: Registro de Ações de Saúde da Atenção Domiciliar; SARGSUS: Sistema de Apoio à Elaboração do Relatório de Gestão; SIA: Sistema de Informações Ambulatoriais; SIAB: Sistema de Informações da Atenção Básica; SIACS: Sistema de Acompanhamento dos Conselhos de Saúde; SIASI: Sistema de Informação da Saúde Indígena; SICLOM: Sistema de Controle e Monitoramento Clínico e Laboratorial dos Pacientes em Tratamento de Hepatite; SIES: Sistema de Informação de Insumos Estratégicos; SIH: Sistema de Informações Hospitalares; SIM: Sistema de Informação sobre Mortalidade; SINAN: Sistemas de Informação de Agravos de Notificação; SINASC: Sistema de Informações sobre Nascidos Vivos; SIS-Água: Sistema de Informação de Vigilância da Qualidade da Água para Consumo Humano; SISCAN: Sistema de Informação do Câncer; SISCEL: Sistema de Controle de Exames Laboratoriais de CD4 e Carga Viral; SIS-CNRAC: Central Nacional de Regulação de Alta Complexidade; SISGENO: Sistema de Controle de Exame de Genotipagem; SISNEO: Sistema Nacional de Triagem Pré-Natal; SIS-PNDC: Sistema do Programa Nacional de Controle de Dengue; SISREG: Sistema Nacional de Regulação; SIS-SOLO: Sistema de Informação de Vigilância em Saúde de Populações Expostas a Solo Contaminado; SISVAN: Sistema de Vigilância e Acompanhamento Nutricional; S IVEP: Sistemas Informatizado de Vigilância Epidemiológica; SNT: Sistema Nacional de Transplante; VETORES-MALÁRIA: Sistema de Informação e Controle de Vetores.

Nota: a partir de 2019, e-SUS Atenção Básica (e-SUS AB) passou a se chamar e-SUS Atenção Primária à Saúde (e-SUS APS).

\section{Discussão}

Um primeiro resultado da pesquisa aponta para um número de SIS de base nacional superior ao relatado na escassa literatura sobre o assunto. Em comparação com o levantamento realizado por Moraes 2, por exemplo, considerado aqui como o mais amplo na produção acadêmica recente, existe uma diferença de 28 sistemas. Quando comparado com outras fontes 3,25 (http://www2.datasus.gov. br/DATASUS/index.php), a diferença é ainda maior, com a ressalva de que nenhum desses estudos se propôs explicitamente a listar a totalidade dos SIS de base nacional no Brasil, mas sim apontar ou analisar aqueles que consideraram mais relevantes.

De uma forma geral, a literatura foca nas bases mais abrangentes e antigas do SUS, os SIS "estruturantes”, tais como Sistema de Informação Ambulatorial (SIA), Sistema de Informações Hospitalares (SIH), Sistema de Informações sobre Nascidos Vivos (SINASC), Sistema de Informação de Agravos de Notificação (SINAN) e Cadastro Nacional de Estabelecimentos de Saúde (CNES), dentre outros. Por outro lado, há uma ausência dos sistemas do DIAHV e alguns sistemas da SVS, tal como SIS-Água, SISSolo, assim como de algumas bases nacionais recentes, como a Autoavaliação das Equipes de Saúde 
da Família (EqSF) do Programa Nacional de Melhoria do Acesso e da Qualidade da Atenção Básica (PMAQ-AB) e o Sistema do Programa Bolsa Família na Saúde.

Um segundo resultado da pesquisa diz respeito à dificuldade do Ministério da Saúde em elaborar uma lista unificada de SIS de base nacional que estão sob sua responsabilidade, acarretando pouca transparência junto ao meio acadêmico e a sociedade de uma forma em geral. Isso decorre, em parte, pela ausência de critérios claros para a definição de um produto de software criado pelo Ministério da Saúde como SIS de base nacional.

Percebeu-se ao longo da pesquisa, todavia, que a própria literatura especializada também não trazia elementos suficientes para uma classificação atualizada desse tipo específico de SIS. Cunha \& Vargens 28 sugerem que sejam definidos como SIS de base nacional os sistemas cujo envio de dados por estados e municípios é obrigatório, porém, diversos SIS de base nacional, especialmente os que não vinculados a políticas de vigilância em saúde e ao controle de repasses federais, eram de uso facultativo.

Pinto et al. 29 (p. 1860) citam como critérios de inclusão o fato de serem de "domínio público, com periodicidade histórica (...) e aqueles que geram dados ou estimativas municipais (pelo menos para as 27 capitais do Brasil)", entretanto, nem todo SIS de base nacional, apesar de estarem disponíveis, são utilizados cotidianamente em todas as capitais, pois este uso pode variar de acordo com perfil epidemiológico de cada região (por exemplo, os Vetores Malária) ou por simples opção do município em não utilizar alguns SIS.

Daniel et al. 30 afirmam que SIS de base nacional se caracterizam como Governo Eletrônico do tipo e-Administração, sem entrar em maiores detalhes.

Diante desse cenário, consideramos necessária uma atualização do conceito de SIS de base nacional que, ao nosso ver, são caracterizados pelo fato de estar (ou ter estado) aberto a receber e armazenar dados de saúde de qualquer região do país, numa base única nacional sob custódia do Estado. Esses dados podem ser enviados por estabelecimentos ou estruturas de gestão da saúde, profissionais de saúde, unidades externas ao SUS (escolas, centros de referência de assistência social, empresas etc.) ou até por cidadãos de forma isolada (no caso de um usuário que registra dados sobre sua saúde, por exemplo).

Um terceiro resultado da pesquisa, relacionado à estrutura de gestão e suporte de TI do SIS aponta para uma significativa rede de núcleos de TI nos maiores departamentos do Ministério da Saúde, alguns criados há décadas, que exercem um papel central no desenvolvimento de sistemas estruturantes do SUS. Apesar disso, esses núcleos e alguns de seus sistemas ainda são quase invisíveis nos documentos e sítios oficiais da política de informação e informática do Ministério da Saúde.

A existência de núcleos de TI não é uma exclusividade do Ministério da Saúde, sendo um fenômeno relativamente comum nas grandes organizações públicas, relatados em documentos oficiais relacionados à governança de TI 31,32,33. Além disso, tal existência não pode ser caracterizada a priori como um problema. Abrão 34 ao analisar a relação entre as áreas de TI de negócios na administração pública federal, descreve pontos positivos e negativos na descentralização da gestão de TI por meio de tais núcleos. Cita como vantagens uma maior apropriação da equipe de TI em relação aos problemas da área técnica, mais agilidade no desenvolvimento de sistemas e melhor antecipação de problemas. Ao mesmo tempo, chama a atenção para o risco de duplicidade de projetos, despadronização tecnológica e a incapacidade do núcleo de TI manter sistemas de grande porte 34 .

Por fim, um último aspecto a ser debatido em relação aos resultados está relacionado à classificação do SIS, nos termos propostos por Moraes 5, em dois grandes troncos genealógicos, ou blocos lógicos, de acordo com os seus principais sentidos de funcionamento, que se mostrou efetiva. De fato, foi possível notar que grande parte dos SIS analisados são caraterizados pela lógica de controle contábil e/ou de monitoramento e controle de populações pelo Estado, e que operam ainda sob intensa fragmentação da informação em saúde, dificultando uma visão integral sobre o indivíduo acompanhado pelos serviços de saúde, e ainda sob uma relação vertical entre o Governo Federal e os outros entes federados.

Porém, como demonstrado, foi detectado que alguns SIS mais recentes não se enquadravam de forma estrita nessas lógicas instituídas. Esses SIS apontam para uma discreta mudança na relação federativa do Governo Federal com estados e municípios, no âmbito da política de TI, que passou a criar e disponibilizar tecnologias cujo principal objetivo é o apoio ao desenvolvimento locorre- 
gional do SUS, com alguma integração aos SIS já existentes. O Sistema de Informação para Atenção Básica (SISAB) e o Prontuário Eletrônico do Cidadão (PEC) da Estratégia e-SUS APS, por exemplo, realizaram uma integração completa com 11 SIS de base nacional em uso na Atenção Básica 35 . O SISREG, um dos maiores SIS de base nacional em uso no Brasil hoje, é utilizado majoritariamente para melhorar o controle e qualidade dos fluxos assistenciais de pacientes entre os serviços de saúde de estados e municípios, não sendo vinculado a políticas de repasse de recursos pelo governo federal ou monitoramento dos pacientes cadastrados em sua base para fins de controle de alguma política nacional de saúde.

\section{Considerações finais}

Para além de identificar os 54 diferentes SIS de base nacional que estiveram em funcionamento no Brasil entre 2010 e 2018 e descrever o portfólio e a estrutura de gestão destes sistemas no Ministério da Saúde, nos propusemos, com base no presente estudo, a suscitar reflexões sobre a própria gestão da Política de TI no âmbito do SUS. O desconhecimento geral sobre a quantidade e a descrição consolidada da lista dos SIS de base nacional é apenas um indicativo da complexidade, da frágil integração e pouca transparência de como tem sido historicamente conduzida a política de TI do Ministério da Saúde. É como se estivéssemos lidando com uma "caixa preta", cujas informações estão muitas vezes inacessíveis a quem opera ou estuda os SIS no país. Não há como se efetivar o controle social sobre aquilo que mal se conhece. Em tempos de Big Data e Machine Learning, a principal ferramenta de acesso e cruzamento dos dados de saúde nacionais é o Tabnet/Tabwin, desenvolvido há quase 30 anos. As regras públicas para acesso às bases nacionais, fundamentais para o desenvolvimento de pesquisas e da gestão nos estados e municípios, ainda carecem de regulamentação específica, principalmente após a aprovação da Lei Geral de Proteção de Dados Pessoais (Lei no 13.709/2018) 36.

O frágil tensionamento produzido por trabalhadores e gestores de saúde e pelo próprio Movimento Sanitário por mais transparência dessas bases, e a pequena produção científica sobre as estruturas e relações de poder envolvidas na gestão do SIS parecem tímidos e insuficientes quando comparados à velocidade de incorporação das TI pelos serviços e gestões da saúde (que ficam cada vez mais dependentes delas para manter o seu funcionamento básico). Em parte, essa dificuldade pode estar relacionada aos desafios de compreensão de um objeto que, devido à confluência de saberes e práticas entre os campos da informação e da informática, vem se tornando mais complexo e demanda estruturas mais significativas de pesquisa multidisciplinar, ainda pouco desenvolvidas no campo da Saúde Coletiva 9.

Parece fazer sentido a aplicação do Enigma da Esfinge a essa relação da Saúde Coletiva com as ITIS - decifrar projetos, interesses, métodos, a micropolítica e a macropolítica desse intercampo é a condição para a garantia do interesse público e de uma regulação eficaz por parte do Estado sobre o mercado, sob pena do setor estatal ser devorado pelas forças econômicas que o cercam. 


\section{Colaboradores}

G. C. Coelho Neto e A. Chioro contribuíram na concepção do projeto, análise dos dados, redação e revisão crítica do artigo e na aprovação final da versão a ser publicada.

\section{Informações adicionais}

ORCID: Giliate Cardoso Coelho Neto (0000-00025580-3586); Arthur Chioro (0000-0001-71842342).

\section{Referências}

1. World Health Organization. Monitoring the building blocks of health systems: a handbook of indicators and their measurement strategies. Geneva: World Health Organization Press; 2010.

2. Moraes IHS. Sistemas de Informação em Saúde: Patrimônio da Sociedade Brasileira. In: Paim JS, Almeira-Filho N, organizadores. Saúde coletiva: teoria e prática. Rio de Janeiro: Editora Medbook; 2014. p. 649-65.

3. Fundação Oswaldo Cruz; Organização Pan -Americana da Saúde. A experiência brasileira em sistemas de informação em saúde. v. 1. Brasília: Editora do Ministério da Saúde; 2009. (Série B. Textos Básicos de Saúde).

4. Conselho Nacional de Secretários de Saúde. Regulação Assistencial e os Sistemas de Informação/Oficina CONASS. Nota técnica no 4, de 27 de maio de 2015. https://www.conass. org.br/wp-content/uploads/2015/07/NT04-2015-REGULAC\%CC\%A7A\%CC\%83OASSISTENCIAL-E-SIS-final.pdf (acessado em Jun/2021).

5. Moraes IHS. Informação em saúde: da prática fragmentada ao exercício da cidadania. São Paulo: Hucitec Editora/Rio de Janeiro: Associação Brasileira de Saúde Coletiva; 1994.

6. Moraes IHS. Política, tecnologia e informação em saúde - a utopia da emancipação. Salvador: Casa da Qualidade Editora; 2002.

7. Campelo F. Sistemas de informação da atenção à saúde: da fragmentação à interoperabilidade. In: Ministério da Saúde, organizador. Sistemas de informação da atenção à saúde: contextos históricos, avanços e perspectivas no SUS. Brasília: Editora Cidade Gráfica e Editoria; 2016. p. 9-21.

8. Araújo YB, Rezende LCM, Queiroga MMD, Santos SR. Sistemas de Informação em Saúde: inconsistências de informações no contexto da atenção primária. J Health Inform 2016; 8(Compl):164-70.

9. Moraes IHS, Gómez MNG. Informação e informática em saúde: caleidoscópio contemporâneo da saúde. Ciênc Saúde Colet 2007; 12:553-65.

10. Departamento de Informática do SUS. DATASUS: trajetória 1991-2002. Brasília: Ministério da Saúde; 2002. (Série G Estatística e Informação em Saúde).
11. Agência Nacional de Transportes Aquaviários. Plano Diretor de Tecnologia da Informação PDTI 2014-2015. Brasília: Agência Nacional de Transportes Aquaviários/Secretaria de Tecnologia da Informação; 2013.

12. Ministério da Saúde. Política Nacional de Informação e Informática em Saúde proposta versão 2.0 (Inclui deliberações da 12a Conferência Nacional de Saúde). http://bvsms.sau de.gov.br/bvs/publicacoes/PoliticaInforma caoSaude29_03_2004.pdf (acessado em 16/ Set/2019).

13. Ministério da Saúde. Portaria no 589, de 20 de maio de 2015. Institui a Política Nacional de Informação e Informática em Saúde (PNIIS). Diário Oficial da União 2015; 22 mai.

14. Ministério da Saúde. Resolução no 6, de 6 novembro de 2013. Dispõe sobre as regras para implantação de novos aplicativos, sistemas de informação em saúde ou novas versões de sistemas e aplicativos já existentes no âmbito do Sistema Único de Saúde (SUS) e que envolvam sua utilização pelo Ministério da Saúde e Secretarias Estaduais, do Distrito Federal e Municipais de Saúde. Diário Oficial da União 2013; 7 nov.

15. Ministério da Saúde. Portaria no 2.073, de 31 de agosto de 2011. Regulamenta o uso de padrões de interoperabilidade e informação em saúde para sistemas de informação em saúde no âmbito do Sistema Único de Saúde, nos níveis municipal, distrital, estadual e federal. Diário Oficial da União 2013; 1 set.

16. Ministério da Saúde. Portaria no 2.072, de 31 de agosto de 2011. Redefine o Comitê de Informação e Informática em Saúde (CIINFO/ MS) no âmbito do Ministério da Saúde. Diário Oficial da União 2011; 1 set.

17. Silva JPL, Travassos CMR, Vasconcellos MM, Campos LM. Revisão sistemática sobre encadeamento ou linkage de bases de dados secundários para uso em pesquisa em saúde no Brasil. Cad Saúde Colet (Rio J.) 2006; 14:197-224.

18. Moreno RA. Interoperabilidade de Sistemas de Informação em Saúde. J Health Inform 2016; 8:I-II.

19. Garde S, Knaup P, Hovenga E, Heard S. Towards semantic interoperability for electronic health records. Methods Inf Med 2007; 46:332-43. 
20. Moraes IHS. Governança e modelo de gestão da informação e inovação. In: Cunha FJAP, Lázaro HBBP organizadores. Conhecimento, inovação e comunicação em serviços de saúde. Salvador: EdUFBA/Rio de Janeiro: Editora Fiocruz; 2014. p. 19-41.

21. de la Torre-Diez I, López-Coronado M, Vaca C, Aguado JS, Castro C. Cost-utility and costeffectiveness studies of telemedicine, electronic, and mobile health systems in the literature: a systematic review. Telemed J E Health 2015; 21:81-5

22. Agha L. The effects of health information technology on the costs and quality of medical care. J Health Econ 2014; 34:19-30.

23. HIMSS Europe. Strategic interoperability in Germany, Spain \& the UK: the clinical and business imperative for healthcare organisations. https://www.himss.eu/content/himsseurope-strategic-interoperability-germanyspain-uk (acessado em 16/Set/2019).

24. Park H, Lee SI, Hwang H, Kim Y, Heo EY, Kim JW, et al. Can a health information exchange save healthcare costs? Evidence from a pilot program in South Korea. Int J Med Inform 2015; 84:658-66.

25. Conselho Nacional das Secretarias Municipais de Saúde. Relatório Final do XXXIII Congresso Nacional de Secretarias Municipais de Saúde. https://www.conasems.org.br/wp-con tent/uploads/2017/09/XXXIIICONASEMSFINAL-1.pdf (acessado em 06/Set/2019).

26. Brasil. Lei no 12.527 , de 18 de novembro de 2011. Regula o acesso a informações previsto no inciso XXXIII do art. 5o, no inciso II do $\$ 3$ o do art. 37 e no $\$ 2$ o do art. 216 da Constituição Federal; altera a Lei no 8.112, de 11 de dezembro de 1990; revoga a Lei no 11.111 , de 5 de maio de 2005, e dispositivos da Lei no 8.159, de 8 de janeiro de 1991; e dá outras providências. Diário Oficial da União 2011; 18 nov.

27. Ministério da Saúde. Portaria no 2.920, de 31 de outubro de 2017. Altera as Portarias de Consolidação n5/GM/MS e 6/GM/MS, de 28 de setembro de 2017, para inclusão do Programa de Informatização das Unidades Básicas de Saúde - PIUBS. Diário Oficial da União 2017; 1 nov.

28. Cunha EM, Vargens JMC. Sistemas de informação do Sistema Único de Saúde. In: Gondim GMM, Christófaro MAC, Miyashiro GM, organizadores. Técnico de vigilância em saúde: fundamentos. Rio de Janeiro: Escola Politécnica de Saúde Joaquim Venâncio, Fundação Oswaldo Cruz; 2017. p. 71-112.
29. Pinto LF, Freitas MPS, Figueiredo AWS. Sistemas Nacionais de Informação e levantamentos populacionais: algumas contribuições do Ministério da Saúde e do IBGE para a análise das capitais brasileiras nos últimos 30 anos. Ciênc Saúde Colet 2018; 23:1859-70.

30. Daniel VM, Pereira GV, Macadar MA. Perspectiva Institucional dos Sistemas de Informação em Saúde em dois estados brasileiros. Revista de Administração Contemporânea 2014; 18:650-69.

31. Instituto Nacional de Pesquisas Espaciais. Plano Diretor de Tecnologia da Informação PDTI/INPE V.06. Biênio 2015-2016. http:// www.inpe.br/ti/arquivos/PDTI-2015_2016. pdf (acessado em 14/Set/2020).

32. Conselho Regional de Engenharia e Agronomia do Rio de Janeiro. PDTI - Plano Diretor de Tecnologia da Informação 2017-2018. https://novoportal.crea-rj.org.br/transparen cia/wp-content/uploads/Plano-Diretor-deTecnologia-da-Informacao-2017-2018.pdf (acessado em 14/Set/2020).

33. Centro Federal de Educação Tecnológica de Minas Gerais. Relatório final - Comitê de Governança e Acesso à Informação. http:// www.sgi.cefetmg.br/wp-content/uploads/si tes/83/2017/08/Relat\%C3\%B3rio-Final-diag n\%C3\%B3stico-TI-2015.pdf (acessado em 14/ Set/2020).

34. Abrão RCS. O relacionamento entre áreas de TI e negócio na administração pública. [Monografia de Conclusão de Curso]. Brasília: Instituto de Ciências Exatas, Universidade Federal de Minas Gerais; 2019.

35. Coelho Neto GC. Integração entre sistemas de informação em saúde: o caso do e-SUS Atenção Básica [Dissertação de Mestrado]. São Paulo: Universidade Federal de São Paulo; 2019.

36. Brasil. Lei no 13.709, de 14 de agosto de 2018. Lei Geral de Proteção de Dados Pessoais (LGPD). Diário Oficial da União 2018; 15 ago. 


\section{Abstract}

The study aimed to identify and analyze the nationwide Health Information Systems (SIS) operating in Brazil from 2010 to 2018 and to understand the management and support structure for Information Technology (IT) in these systems in the Ministry of Health. This was a descriptive study based on document analysis, seeking to consolidate the data on nationwide SIS based on selection criteria referenced on the concept of SIS. The documents yielded incomplete and mutually disparate data, evidencing different interpretations of the concepts of systems and health information systems in the Ministry of Health. The survey found 54 nationwide Health Information Systems functioning from 2010 to 2018 and collected information on seven decentralized IT centers in Ministry of Health departments, which work autonomously from the Brazilian Health Informatics Department (DATASUS). The analysis of the logics of their functioning identified four groups of SIS pertaining to the following: monitoring relevant events for public health, greater control and monitoring of health programs in the Ministry of Health, registration of physical and legal persons, and a "new generation" of SIS that are developed and maintained by the Federal Government but differ from its verticalized programmatic logic, focused on administrative rationalization and improving the quality of services provided by the SUS at the local and regional levels.

Primary Health Care; Health Information Systems; Information Technology; Systems Integration

\section{Resumen}

El objetivo de la investigación fue identificar y analizar los Sistemas de Información en Salud (SIS) de base nacional, que estuvieron en funcionamiento en Brasil entre los años de 2010 y 2018, así como comprender la estructura de gestión y soporte de Tecnología de la Información (TI) de estos sistemas en el Ministerio de Salud. Se trata de un estudio descriptivo, basado en análisis documental, donde se buscó consolidar datos sobre los SIS de extensión nacional, a partir de criterios de selección referidos al concepto de SIS. Los documentos analizados presentaron datos incompletos y bastante diferentes entre sí, evidenciando las diferentes interpretaciones de los conceptos de sistemas y sistemas de información de salud en el Ministerio de Salud. Se encontraron 54 SIS de base nacional, en funcionamiento durante el periodo de 2010 a 2018, y se recogió información sobre siete núcleos de TI descentralizados en departamentos del Ministerio de Salud, que trabajan de forma autónoma del Departamento de Informática del Sistema Único de Salud (DATASUS). Al analizar sus lógicas de funcionamiento, se identificaron cuatro grupos de SIS: un primero, relativo al monitoreo de eventos de relevancia para la salud pública, un segundo, dirigido hacia un mayor control y supervisión de programas de salud del Ministerio de Salud, un tercero, dedicado al registro general de personas físicas y jurídicas y un cuarto que puede ser considerado una "nueva generación" de SIS puesto que, a pesar de que hayan sido desarrollados y mantenidos por el Gobierno Federal, se desprenden de una lógica programática verticalizada, destinándose a la racionalización administrativa y mejora de la calidad de la prestación de servicios del SUS en el ámbito locorregional.

Atención Primaria de Salud; Sistemas de Información en Salud; Tecnología de la Información; Integración de Sistemas
Recebido em 19/Set/2019

Versão final reapresentada em 15/Set/2020

Aprovado em 23/Nov/2020 